\title{
Suplementación probiótica con Lactobacillus casei en cerdas y su efecto sobre los parámetros zootécnicos de los lechones
}

\author{
Probiotic supplementation with Lactobacillus casei in sows and its effects on the \\ zootechnical parameters of piglets
}

\author{
Rafael Suárez ${ }^{1,2,4}$, Natalia Buitrago ${ }^{1}$, Iang Rondón-Barragán ${ }^{2,3}$
}

\section{Resumen}

\begin{abstract}
El objetivo del estudio fue evaluar la suplementacion de Lactobacillus casei en cerdas y su efecto sobre los parámetros zootécnicos de los lechones. Se trabajo con 18 cerdas F1 Landrace $\mathrm{x}$ Large White distribuidas en tres grupos completamente al azar: control y dos grupos suplementados con L. casei (T1: $10 \times 10^{6} \mathrm{UFC}$ [unidades formadoras de colonias]; T2: $10 \times 10^{8}$ UFC). Las dosis fueron administradas semanalmente por vía oral $2 \mathrm{~h}$ después de la primera alimentación del día (09:00) durante 180 días, cubriendo un ciclo productivo. Se analizaron los resultados de las variables zootécnicas de cada hembra y sus lechones al periparto, al destete y al nuevo servicio de cubrición de la hembra. En el periparto se encontró un mayor peso promedio de nacidos totales (PPNT) y peso promedio de nacidos vivos (PPNV) en los animales suplementados en comparación con el grupo control $(\mathrm{p}<0.05)$. En la fase de lactancia se evidenció mayor peso total por camada al destete (PTCD), peso promedio lechón desteto (PPLD), ganancia total por camada desteta por hembra (GTCDH), ganancia total promedio por lechón, (GTPL) y ganancia promedio por lechón día (GPLD) en los animales suplementados con el probiótico comparado con el grupo control $(\mathrm{p}<0.01)$. En ninguna de las variables se evidenció diferencias entre los grupos suplementados. Se concluye que la suplementación oral con el probiótico Lactobacillus casei en las cerdas en fase de gestación y lactancia mejora los índices productivos en el periodo periparto y en la fase de lactancia de los lechones.
\end{abstract}

Palabras clave: probiótico; Lactobacillus casei; cerdos; suplementación

${ }^{1}$ Grupo de Investigación en Genética y Biotecnología de la Reproducción, Facultad de Medicina Veterinaria y Zootecnia, Universidad del Tolima, Ibagué, Tolima, Colombia

${ }^{2}$ Grupo de Investigación en Inmunobiología y Patogénesis, Facultad de Medicina Veterinaria y Zootecnia, Universidad del Tolima, Ibagué, Tolima, Colombia

${ }^{3}$ Grupo de Investigación en Avicultura, Facultad de Medicina Veterinaria y Zootecnia, Universidad del Tolima, Ibagué, Tolima, Colombia

${ }^{4}$ E-mail: rasuarezm@ut.edu.co

Recibido: 20 de septiembre de 2018

Aceptado para publicación: 23 de febrero de 2019 
The aim of this study was to evaluate the supplementation of Lactobacillus casei in sows and its effect on the zootechnical parameters of piglets. A total of 18 F1 Landrace $\mathrm{x}$ Large White sows were distributed in three completely randomized groups: control and two groups supplemented with L. casei (T1: 10 x 10 $\mathrm{CFU}$ [colony forming units]; T2: 10 $\left.\mathrm{x} 10^{8} \mathrm{CFU}\right)$. The doses were administered weekly orally $2 \mathrm{~h}$ after the first feeding of the day (09:00) for 180 days, covering a productive cycle. Results of zootechnical variables of each female and her piglets at the peripartum, at weaning and at the new service of the female were evauated. In the peripartum, a greater mean body weight of total births (PPNT) and average birth body weight were found (PPNV) in the supplemented animals compared to the control group $(\mathrm{p}<0.05)$. In the lactation phase there was a greater total weight per litter at weaning (PTCD), mean body weight per piglet at weaning (PPLD), total body weight gain per litter per sow (GTCDH), mean total body weight per piglet (GTPL) and mean body weight per day pero piglet (GPLD) in the animals supplemented with the probiotic compared to the control group $(p<0.01)$. None of the variables showed differences between the supplemented groups. It is concluded that oral supplementation with the probiotic Lactobacillus casei in the sows during the gestation and lactation phase improves the productive indexes in the peripartum period and in the lactation phase of the piglets.

Key words: probiotic; Lactobacillus casei; pigs; supplementation

\section{INTRODUCCIÓN}

La necesidad de maximizar la productividad de los sistemas pecuarios llevaron al uso de antibióticos a niveles subterapéuticos como promotores de crecimiento para mejorar la utilización de los nutrientes (Enrique et $a l ., 2005)$ y disminuir la incidencia de algunas enfermedades (Williams et al., 2001; Giraldo et al., 2015). Sin embargo, el uso excesivo e inadecuado de antibióticos ha creado una enorme presión selectiva en las poblaciones bacterianas, propiciando la aparición de cepas multiresistentes capaces a su vez de transferir dicha resistencia (Mathur y Singh, 2005). Así mismo, se han demostrado genes de resistencia idénticos en diversas especies bacterianas de diferentes hospederos (Scott, 2002; Pantozzi et al., 2010; Ibar, 2017), lo cual genera preocupación a nivel mundial por el potencial riesgo de transferencia de la resistencia entre la microbiota animal y humana (Mathur y Singh, 2005; Esperbent y Migliorati, 2017). De esta ma- nera, la Unión Europea determinó la prohibición del uso de antibióticos con fines de promoción del crecimiento desde el 1 de enero de 2006 (European Parliament, 2003).

Diversas alternativas al uso de antibióticos en animales de producción han sido propuestas, incluyendo prebióticos, probióticos, enzimas, sustancias húmicas y $\beta$-glucanos, entre otras, tendientes a estimular la inmunidad y a mejorar de los parámetros productivos (Piñeros et al., 2012; Li et al., 2017). En cerdos, el uso de probióticos ha demostrado mejorar los parámetros productivos en diversas fases de la producción (Lázaro et al., 2005). Adicionalmente, se ha evidenciado que el uso de Bifidobacterium lactis HN019 y Enterococcus faecium disminuyen la presentación de diarreas en lechones asociadas a rotavirus y E. coli (Shu et al., 2001; Taras et al., 2006); efecto que ha sido ademas demostrado con bacterias como Lactobacillus gasseri, L. reuteri, L. acidophilus y $L$. fermentum (Huang et al., 2002). 
Teniendo en cuenta los efectos de los probióticos sobre la fisiología intestinal, se hace necesario aplicarlos en los puntos críticos de la producción porcina, buscando beneficios zootécnicos para el productor. Los periodos de gestación, lactancia y destete constituyen puntos criticos en la producción debido a los cambios fisiológicos en la hembra para suplir los requerimientos de los lechones. Se ha demostrado que las bacterias probióticas tienen la facultad de ser transferidas desde la cerda a los lechones por contacto con las heces, antes de que estos inicien el consumo de alimento sólido (Taras et al., 2005; Giraldo et al., 2015), lo cual promueve el desarrollo de la inmunidad intestinal y la actividad proliferativa de las cripas (Giraldo et al., 2015), aumentando así la densidad de la vellosidad intestinal (Enrique et al., 2005; Castillo et al., 2007) que da inicio a la actividad fermentativa del colon mediante los componentes provenientes de la dieta (Casewell et al., 2003), favoreciendo el crecimiento y desarrollo del lechón. El presente estudio tuvo como objetivo evaluar los parámetros zootécnicos de lechones hijos de cerdas suplementadas con probiótico L. casei a través de un ciclo productivo.

\section{Materiales y Métodos}

\section{Animales Experimentales}

Los experimentos fueron conducidos en la Central Reproductiva Porcigán (Cajamarca, Tolima, Colombia). Se emplearon 18 cerdas clínicamente sanas, F1 Large White $\mathrm{x}$ Landrace, con edad productiva de tres partos. Los animales contaron con planes sanitarios completos, según el régimen normal de producción (vermifugación a los 80 días, Doramectina) y vacunación a los 10 días posparto contra erisipela, parvovirus y seis serovares de Leptospira spp (Zoetis, Argentina). Los animales fueron alimentados con una dieta comercial y sostenidos bajo las mismas condiciones ambientales, con temperatura promedio de $18{ }^{\circ} \mathrm{C}$. El estudio fue ava- lado por el Comité Local de Ética siguiendo la normatividad de la Ley 84 de 1989, Resolución 8430 de 1993 para el uso de animales vivos, y bajo los lineamientos del Código de Ética para el ejercicio profesional de la medicina veterinaria, la medicina veterinaria y zootecnia y zootecnia (Ley 576 de 2000).

\section{Probiótico Lactobacillus casei}

Como cepa probiótica se empleó Lactobacillus casei (ATCC ${ }^{\circledR} 393^{\mathrm{TM}}$ ) obtenida de una casa comercial (ATCC ${ }^{\circledR}$, USA) y almacenada a $-20{ }^{\circ} \mathrm{C}$ en caldo De Man, Rogosa y Sharpe (MRS) (Oxoid, Alemania) suplementado con $20 \%$ de glicerol (v/v). Las alícuotas de la bacteria se reactivaron en caldo MRS con incubación a $37^{\circ} \mathrm{C}$ durante 24 $\mathrm{h}$, y luego fueron sembradas en agar MRS e incubadas a $37^{\circ} \mathrm{C}$ durante $48 \mathrm{~h}$. Las colonias aisladas se transfirieron a caldo MRS (Oxoid, Alemania) e incubaron a $37^{\circ} \mathrm{C}$ hasta que alcanzaron una turbidez similar al tubo 1 en la escala de MacFarland ( $\left.3 \times 10^{8} \mathrm{UFC} / \mathrm{ml}\right)$. Se realizaron diluciones seriadas (1:10) en $\mathrm{NaCl} 0.85 \%$ (w/v) obteniendo las concentraciones de $10 \times 10^{6}$ y $10 \times 10^{8}$ UFC (Colombo et al., 2014).

\section{Suplementación con $L$. casei}

Los sujetos experimentales fueron distribuidos en tres grupos completamente al azar, un grupo control $(\mathrm{n}=6), \mathrm{T} 1(\mathrm{n}=6)$ suplementado con $10 \times 10^{6}$ UFC de L. casei, y T2 suplementado con $10 \times 10^{8}$ UFC de L. casei. El inóculo $(20 \mathrm{ml})$ fue administrado semanalmente vía oral mediante una jeringa dosificadora por un periodo de 180 días, cubriendo más de un ciclo productivo completo, incluyendo el proceso de adaptación.

Durante la gestación, la alimentación fue restringida hasta el día 85 recibiendo 2 $\mathrm{kg}$ /día de alimento comercial gestación y del día 86 al 114 se suministró $3 \mathrm{~kg} /$ día de alimento comercial de lactancia. Posterior al parto, las cerdas iniciaron consumiendo $1 \mathrm{~kg}$ /día y aumentaron de manera gradual hasta alcanzar $6.5 \mathrm{~kg}$ /día. Los lechones al nacer se maneja- 
ron siguiendo los protocolos y procedimientos estandarizados por la granja. Los lechones fueron pesados en una balanza colgante digital WeiHeng ${ }^{\circledR}$ de referencia WH-C100 al nacer y a las 24 horas de vida para homogenizar el peso de los grupos, buscando un peso promedio similar y un mismo número de lechones a lactar. Cada grupo de tratamiento contó con 66 lechones $(11 \mathrm{de}$ promedio por hembra) para un total de 198 lechones evaluados. Se respetó el intercambio de lechones dentro de los mismos grupos. Los lechones sobrantes fueron atetados y retirados del experimento. Los lechones fueron nuevamente pesados a los 21 días.

\section{Evaluación Zootécnica}

Los parámetros productivos evaluados en las camadas incluyeron: NT: nacidos totales; PTNT: peso total de nacidos totales; PPNT: peso promedio de nacidos totales; NV: nacidos vivos; PTNV: peso total de nacidos vivos; PPNV: peso promedio de nacidos vivos; MU: muertos; MO: momias; MTP: mortalidad total al periparto; DUP: duración del parto; PTCI: peso total camada inicial (24 horas pos-nacimiento); PPIL: peso promedio inicial lechón (24 h pos-nacimiento); D: lechones destetos; PTCD: peso total por camada al destete; PPLD: peso promedio lechón desteto; GTCDH: ganancia total por camada desteta por hembra; GTPL: ganancia total promedio lechón; GPLD: ganancia promedio por lechón por día; MUL: lechones muertos en lactancia; RCPP: retorno de celo posparto; PTD: peso total destetos.

\section{Análisis Estadístico}

Los datos fueron analizados mediante SPSS v. 22 para Windows (IBM, EEUU), sometidos a un análisis descriptivo unidimensional y posterior a la validación de los supuestos estadísticos, analizados mediante el análisis de varianza, seguido de comparación múltiple de Tukey o Dunnett, como análisis pos hoc, según cada caso. La prue- ba de Kruskal-Wallis fue empleada para variables no paramétricas. En el análisis de la variable peso de lechones al nacimiento se empleó un modelo de correlación de Pearson entre el tamaño de camada y peso de lechones al nacimiento. Las diferencias fueron consideradas significativas con un valor de $\mathrm{p}<0.05$.

\section{Resultados y Discusión}

Durante el periodo experimental, los animales no mostraron signos clínicos compatibles con patologías o condiciones clínicas que pudiesen influir negativamente los resultados. Los parámetros zootécnicos de los animales suplementados con L. casei así como del grupo control, evaluados en el periodo periparto son descritos en el Cuadro 1 $\mathrm{y}$ aquellos de la fase de lactancia en el Cuadro 2 .

El peso promedio de nacidos totales (PPNT) y peso promedio de nacidos vivos (PPNV) fueron mayores en los animales suplementados con $L$. casei que en el grupo control $(\mathrm{p}<0.05)$ (Figura 1), coincidiendo con Lázaro et al. (2005). No obstante, no se evidenciaron diferencias entre los dos grupos suplementados con el probiótico. Así mismo, Taras et al. (2005) suplementaron cerdas con Bacillus cereus encontrando diferencias significativas en el PPNV, y Guerra et al. (2007) suplementando con L. casei obtuvieron una mejor respuesta productiva; no obstante, la suplementacion con Enterocuccus faecium como probiótico en el estudio de Taras et al. (2006) no indujo tales diferencias en el PPNV. La variabilidad en estos resultados entre estudios puede deberse a la capacidad de la cepa probiótica para ejercer un efecto benéfico, asi como a la influencia de factores nutricionales y ambientales, que controlan la flora microbiana y, por consiguiente, el metabolismo energético para desarrollar mayor crecimiento de los fetos (García et al., 2005; Cesária et al., 2012; Heinritz et al., 2016). 
Cuadro 1. Parámetros zootécnicos de lechones en la fase del periparto provenientes de hembras suplementadas con Lactobacillus casei

\begin{tabular}{cccccc}
\hline Grupo & $\begin{array}{c}\text { NT } \\
(\mathrm{n})\end{array}$ & $\begin{array}{c}\text { PTNT } \\
(\mathrm{kg})\end{array}$ & $\begin{array}{c}\text { PPNT } \\
(\mathrm{g})\end{array}$ & $\begin{array}{c}\text { NV } \\
(\mathrm{n})\end{array}$ & $\begin{array}{c}\text { PTNV } \\
(\mathrm{kg})\end{array}$ \\
\hline & & & $*$ & & \\
Control & $12.5 \pm 1.5^{\mathrm{a}}$ & $16.1 \pm 1.8^{\mathrm{a}}$ & $1374.5 \pm 167.9^{\mathrm{a}}$ & $11.2 \pm 1.2^{\mathrm{a}}$ & $15.1 \pm 2.2^{\mathrm{a}}$ \\
T1 & $11.0 \pm 1.1^{\mathrm{a}}$ & $17.8 \pm 1.2^{\mathrm{a}}$ & $1626.3 \pm 182.7^{\mathrm{b}}$ & $10.3 \pm 0.8^{\mathrm{a}}$ & $16.8 \pm 2.1^{\mathrm{a}}$ \\
T2 & $11.5 \pm 1.1^{\mathrm{a}}$ & $18.7 \pm 1.0^{\mathrm{a}}$ & $1635.0 \pm 158.0^{\mathrm{b}}$ & $10.8 \pm 1.0^{\mathrm{a}}$ & $17.6 \pm 1.3^{\mathrm{a}}$ \\
\hline & $\mathrm{PPNV}$ & $\mathrm{MU}$ & $\mathrm{MO}$ & $\mathrm{MTP}$ & DUP \\
& $(\mathrm{g})$ & $(\%)$ & $(\%)$ & $(\%)$ & $($ horas$)$ \\
& $*$ & & & & $* *$ \\
Control & $1434.6 \pm 136.7^{\mathrm{a}}$ & $5.0^{\mathrm{a}}$ & $5.1^{\mathrm{a}}$ & $10.1^{\mathrm{a}}$ & $02: 45 \pm 00: 38^{\mathrm{a}}$ \\
T1 & $1657.2 \pm 138.7^{\mathrm{b}}$ & $4.2^{\mathrm{a}}$ & $1.4^{\mathrm{a}}$ & $5.6^{\mathrm{a}}$ & $03: 47 \pm 00: 36^{\mathrm{ab}}$ \\
T2 & $1653.4 \pm 149.2^{\mathrm{b}}$ & $5.6^{\mathrm{a}}$ & $0.0^{\mathrm{a}}$ & $5.6^{\mathrm{a}}$ & $04: 35 \pm 01: 14^{\mathrm{b}}$ \\
\hline
\end{tabular}

$\mathrm{a}, \mathrm{b}$ Letras diferentes dentro de cada parámetros zootécnico indican diferencias significativas en la prueba de Tukey: ${ }^{*} p<0.05 ;{ }^{* *} p<0.01$

Cuadro 2. Parámetros zootécnicos de lechones en la fase de lactancia provenientes de hembras suplementadas con Lactobacillus casei

\begin{tabular}{lcccccc}
\hline Grupo & $\begin{array}{c}\text { PTCI } \\
(\mathrm{kg})\end{array}$ & $\begin{array}{c}\text { PPIL } \\
(\mathrm{g})\end{array}$ & $\begin{array}{c}\mathrm{D} \\
(\mathrm{n})\end{array}$ & $\begin{array}{c}\text { PTCD } \\
(\mathrm{kg})\end{array}$ & $\begin{array}{c}\text { PPLD } \\
(\mathrm{g})\end{array}$ & $\begin{array}{c}\text { GTCDH } \\
(\mathrm{kg})\end{array}$ \\
\hline & & & & $* *$ & $* *$ & $* *$ \\
Control & $18.99 \pm 2.0^{\mathrm{a}}$ & $1726.7 \pm 182.3^{\mathrm{a}}$ & $10.2 \pm 0.8^{\mathrm{a}}$ & $62.1 \pm 3.5^{\mathrm{a}}$ & $6134.3 \pm 469.9^{\mathrm{a}}$ & $43.2 \pm 4.0^{\mathrm{a}}$ \\
T1 & $18.97 \pm 2.0^{\mathrm{a}}$ & $1724.6 \pm 177.4^{\mathrm{a}}$ & $10.50 \pm 0.8^{\mathrm{a}}$ & $71.6 \pm 3.9^{\mathrm{b}}$ & $6834.3 \pm 323.8^{\mathrm{b}}$ & $52.6 \pm 5.1^{\mathrm{b}}$ \\
$\mathrm{T} 2$ & $18.92 \pm 1.9^{\mathrm{a}}$ & $1719.9 \pm 171.5^{\mathrm{a}}$ & $10.50 \pm 0.8^{\mathrm{a}}$ & $73.5 \pm 5.4^{\mathrm{b}}$ & $7007.4 \pm 164.6^{\mathrm{b}}$ & $54.6 \pm 4.2^{\mathrm{b}}$ \\
\hline & $\mathrm{GTPL}$ & GPLD & PTD & MUL & RCPP & \\
& $(\mathrm{g})$ & $(\mathrm{g})$ & $(\mathrm{kg})$ & $(\%)$ & $($ día $)$ & \\
& $* *$ & $* *$ & $* *$ & & & \\
Control & $4248.5 \pm 297.9^{\mathrm{a}}$ & $202.3 \pm 14.2^{\mathrm{a}}$ & $62.1 \pm 3.5^{\mathrm{a}}$ & $7.6^{\mathrm{a}}$ & $6.7 \pm 0.8^{\mathrm{a}}$ & \\
T1 & $5016.8 \pm 365.9^{\mathrm{b}}$ & $238.9 \pm 17.4^{\mathrm{b}}$ & $71.6 \pm 3.9^{\mathrm{b}}$ & $4.5^{\mathrm{a}}$ & $7.8 \pm 1.2^{\mathrm{a}}$ & \\
T2 & $5204.1 \pm 177.7^{\mathrm{b}}$ & $247.8 \pm 8.5^{\mathrm{b}}$ & $73.5 \pm 5.4^{\mathrm{b}}$ & $4.5^{\mathrm{a}}$ & $8.8 \pm 3.2^{\mathrm{a}}$ & \\
\hline
\end{tabular}

a,b Letras diferentes dentro de cada parámetros zootécnico indican diferencias significativas en la prueba de Tukey: ${ }^{*} p<0.05 ;{ }^{* *} p<0.01$

El número de NT y NV mostraron una correlación positiva de $0.954(\mathrm{p}<0.01)$. Adicionalmente, los NT mostraron una correlación negativa de -0.77 con PPNT y -0.66 con PPNV, indicando que a mayor tamaño de camada, menores son los pesos promedios de los NT. Resultados similares fueron obtenidos por Lázaro et al. (2005) al suplementar con Saccharomyces cerevisiae. Sin embargo, Jurgens et al. (1997) usando el mismo probiótico en cerdas no encontraron diferencias en el PPNT al nacimiento.

En cerdas gestantes se han utilizado probióticos con el fin de mejorar la cantidad y calidad de las camadas, así como la reduc- 
PPNT

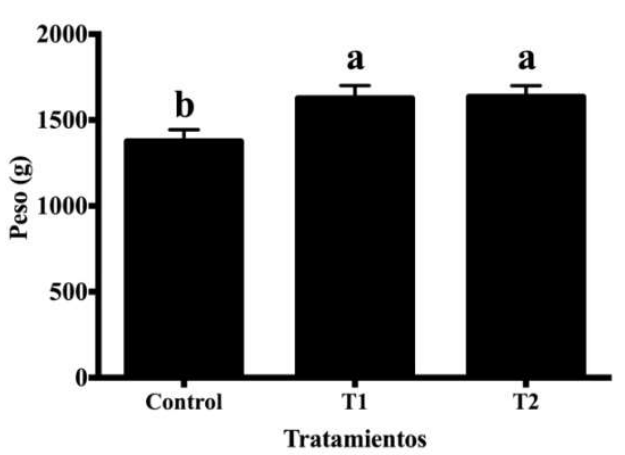

PPNY

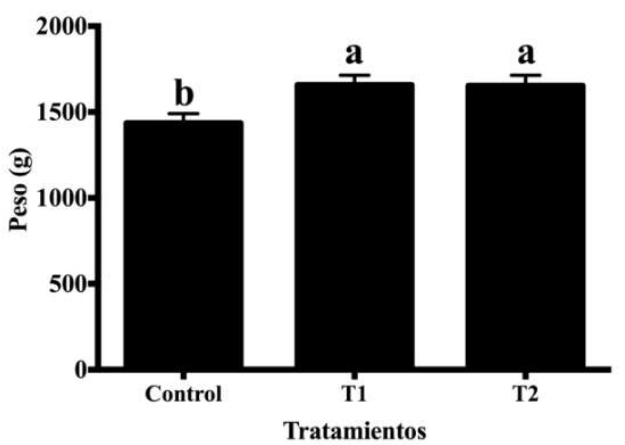

Figura 1. Valores de peso promedio de nacidos totales (PPNT) y peso promedio de nacidos vivos (PPNV) en lechones provenientes de cerdas de tercer parto suplementadas con L. casei. Letras diferentes representan diferencias significativas entre tratamientos

ción de mortinatos; sin embargo, los resultados no son concluyentes. Así, en los trabajos de Taras et al. $(2005,2007)$ se evidenciaron mejoras significativas en el desempeño de las hembras al ser suplementadas con probióticos, efectos que no fueron evidentes en estudios con condiciones similares (Taras et al., 2006). Por lo tanto, se hace necesario profundizar en las interacciones entre organismos, dosis, frecuencias, ambiente y vías de suministro.

La variable MTP fue de $10.1 \%$ en el control, mayor que en $\mathrm{T} 1$ y T 2 con $5.6 \%$ para ambos casos. Si bien estas diferencias no fueron estadísticamente significativas, $L$. casei pudo haber tenido algún efecto en el total de nacimientos, siendo estos valores totalmente influenciados por el número de momias presentadas en los partos donde de presentó 5.1, 1.4 y $0 \%$ para el control, $\mathrm{T} 1$ y T2, respectivamente. El valor de MTP reportado por Diaz et al. (2011) como referencia para Colombia es de $3.8 \%$ y para momias es de $3.5 \%$, los cuales están por debajo y por encima de los grupos del experimento, respectivamente.
La duración del parto presentó diferencias entre tratamientos $(p<0.01)$, donde las cerdas en $\mathrm{T} 2$ evidenciaron tiempos de parto mayores que el grupo control. No obstante, se debe considerar que el efecto del PPNT pudo haber tenido influencia, ya que las hembras pueden presentar mayor dificultad al parto cuando los lechones tienden a ser de mayor tamaño (Huerta, 2010).

Los parámetros zootécnicos de producción de los lechones hijos de hembras suplementadas con probióticos mostraron diferencias entre los tratamientos para las variables PTCD, PPLD, GTCDH, GTPL, GPLD y PTD, siendo los mayores valores para los animales suplementados con respecto al grupo control, pero sin evidenciar diferencias entre los grupos suplementados. Los resultados de PPLD del presente estudio concuerda con el estudio de Quemac (2014), quienes demostraron mayores pesos de lechones al nacimiento, provenientes de hembras suplementadas en las etapas de preparto y lactancia. En el estudio de Ross et al. (2010), el suministro de probiótico a lechones en dosis de $10^{8} \mathrm{UFC} / \mathrm{ml}$ mostró que el grupo tratado 
exhibió menor consumo de alimento y mayor eficiencia en la ganancia de peso que el grupo control. Un meta-análisis de los datos de varios experimentos controlados aleatoriamente mostró que la suplementación con probióticos incrementó la ganancia diaria de peso y mejoró la conversión en los lechones (Zimmermann et al., 2016). Por otro lado, Mejía et al. (2007) no encontró difererencias en lechones en el PPLD ni en la GPLD entre grupos tratados con probióticos de tipo comercial $o$ artesanal.

El PTCI y PPIL no presentaron diferencias entre tratamientos (Cuadro 2), siendo esto una evidencia de homogeneidad, generada para el análisis de las variables zootécnicas en la fase de lactancia, al inicio del experimento. Tampoco se encontraron diferencias en el número de lechones destetos por hembra $(10.2,10.5$ y 105 para el control $\mathrm{T} 1$ y $\mathrm{T} 2$, respectivamente \pm 0.84 ), lo cual estuvo asociado a las tasas de mortalidad de cada grupo experimental (7.6, 4.5 y $4.5 \%$ para el control, T1 y T2, respectivamente), estando el grupo control ceracano al 7\% reportado por Huerta (2010). Se ha demostrado que hembras lactantes suplementadas con bacterias probióticas transfieren microorganismos a los lechones lactantes antes de la alimentación balanceada (preiniciación disponible a los 15 días), induciendo la reducción significativa de la diarrea posdestete (Taras et al., 2007).

En un estudio de suplementación con probióticos realizado por Lázaro et al. (2005) se encontró un mayor peso al nacimiento y su correlación con el tamaño de la camada, comparado con animales no suplementados. No obstante, en dicho estudio, el peso al destete de los lechones del grupo suplementado con probiótico y los del grupo control fueron estadísticamente similares, lo cual difiere de lo reportado en el presente estudio donde la ganancia de peso fue mayor en los grupos suplementados con probióticos con respecto al grupo control. Así mismo, el presente estudio coincide con Bohmer et al. (2006) y Kritas et al. (2015), quienes demostraron que las hembras suplementadas con Enterococcus faecium y Bacillus subtilis, respectivamente, destetaron lechones significativamente más pesados que los alimentados con la dieta de control ( $p<0.05)$, y esto probablemente asociado a que las hembras suplementadas comieron más alimento durante la lactancia $(p<0.05)$. Adicionalmente, estas diferencias pueden deberse a la producción y liberación de enzimas hidrolíticas por parte de los probióticos, favoreciendo la digestión de los alimentos, mejorando el metabolismo de los lípidos y el equilibrio de la flora del tracto gastrointestinal del hospedero, lo cual favorece la ganancia de peso (García et al., 2005; Ortiz y Reuto, 2007).

La suplementación con L. casei tuvo una respuesta variable para diferentes parámetros zootécnicos, de los cuales mostró beneficios productivos en los lechones. En el periparto mostró mayor peso promedio al nacimiento, mayor PPNT y PPNV, y en la fase de lactancia mostró valores mayores de PTCD, PPLD, GTCDH y GTPL.

\section{Conclusiones}

- La suplementación oral de las cerdas con el probiótico Lactobacillus casei mostró mejores resultados en los pesos al nacimiento y al destete comparada con el grupo control. Así mismo, durante la fase de lactancia evidenció mayor ganancia de peso en los lechones

- El uso de probióticos como suplemento nutricional en cerdas mejora los parámetros productivos en la fase de periparto y la lactancia y se vislumbra como una alternativa al uso de antibióticos como promotores de crecimiento en la producción porcina. 


\section{Literatura Citada}

1. Böhmer B, Kramer W, Roth-Maier D. 2006. Dietary probiotic supplementation and resulting effects on performance, health status, and microbial characteristics of primiparous sows. J Anim Physiol Anim Nutr (Berl) 90: 309-315. doi:10.1111/j.1439-0396.2005.-00601.x

2. Casewell M, Friis C, Marco $E$, Mcmullin P, Phillips I. 2003. The European ban on growth-promoting antibiotics and emerging consequences for human and animal health. J Antimicrob Chemoth 52: 159-161. doi: 10.1093/jac/dkg313

3. Castillo M, Martýn S, Manzanilla E, Gasa J. 2007. Changes in caecal microbiota and mucosal morphology of weaned pigs. Vet Microbiol 124: 239247. doi: 10.1016/j.vetmic.2007.04.026

4. Cesária T, Escobar K, Magné A, Mariscal G, Aguilera A. 2012. Nutritional changes in piglets and morphophysiologic development of their digestive tract. Vet México 43:155-173.

5. Colombo $M$, de Oliveira $A E$, de Carvalho AF. Nero LA. 2014. Development of an alternative culture medium for the selective enumeration of Lactobacillus casei in fermented milk. Food Microbiol 39: 89-95. doi: 10.1016/ j.fm.2013.11.008

6. European Parliament. 2003. Regulation (EC) No 1831/2003 of the European Parliament and of the Council of 22 September 2003 on additives for use in animal nutrition. Official J European Union L268:29.

7. Diaz C, Rodríguez M, Vera V, Ramírez G, Casas G, Mogollon J. 2011. Characterization of pig farms in the main swine producing regions of Colombia. Rev Colomb Cienc Pec 24: 131-144.

8. Enrique F, Budiño L, Thomaz M, Kronka R, Satiko L, Nakaghi O, Tucci $\boldsymbol{F}$, et al. 2005. Effect of probiotic and prebiotic inclusion in weaned piglet diets on structure and ultra-structure of small intestine. Braz Arch Biol Techn 48: 921 929. doi: 10.1590/S1516-89132005000800008

9. Esperbent C, Migliorati M. 2017. Bacterias multirresistentes: una amenaza oculta que crece. Rev Invest Agropec 43: 6-10.

10. García Y, García Y, López A, Boucourt R. 2005. Probióticos: una alternativa para mejorar el comportamiento animal. Cuban J Agr Sci 39: 129-140.

11. Giraldo-Carmona J, Narváez W, Díaz E. 2015. Probiotics in swine: contradictory results. Biosalud: 14: 8190. doi: 10.17151/biosa.2015.14.1.9

12. Guerra N, Fajardo P, Mendez J, Cachaldora P, Castro P. 2007. Production of four potentially probiotic lactic acid bacteria and their evaluation as feed additives for weaned piglets. Anim Feed Sci Tech 134: 89-107. doi:10.1016/j.anifeedsci.2006.05.010

13. Heinritz S, Weiss E, Eklund M, Aumiller T, Louis S. 2016. Intestinal microbiota and microbial metabolites are changed in a pig model fed a high-fat/ low-fiber or a low-fat/high-fiber diet. Anim Welfare 11: e0154329. doi: 10.1371/journal.pone.0154329

14. Huang C, Qiao S, Li D, Piao X, Ren J. 2002. Effects of lactobacilli on the performance, diarrhea incidence, vfa concentration and gastrointestinal microbial flora of weaning pigs. Asian Austral J Anim 17: 401-409. doi: 10.5713/ ajas. 2004.401

15. Huerta H. 2010. Determinación de tres parámetros técnicos, mediante monitoreos semanales en granjas porcinas del estado de Veracruz. Tesis de Médico Veterinario Zootecnista. Torreón, México: Univ. Autónoma Agraria Antonio Narro. 47 p.

16. Ibar M. 2017. Salmonella en cerdos: serovariedades y aspectos de la resistencia antimicrobiana relacionados con la Salud Pública en cepas aisladas en granjas y en animales faenados. Tesis de Doctorado. Argentina: Univ. Nacional de la Plata. 193 p. 
17. Jurgens MH, Rikabi RA, Zimmerman DR. 1997. The effect of dietary active dry yeast supplement on performance of sows during gestation-lactation and their pigs. J Anim Sci: 75: 593-597. doi: 10.2527/1997.753593x

18. Kritas S, Marubashi T, Filioussis G, Petridou E, Christodoulopoulos G, Burriel A, Pískoriková M. 2015. Reproductive performance of sows was improved by administration of a sporing bacillary probiotic (Bacillus subtilis C3102). J Anim Sci: 93: 405-413. doi:10.2527/jas.2014-7651

19. Lázaro C, Carcelén F, Torres M, Ara M. 2005. Efecto de probióticos en el alimento de marranas sobre los parámetros productivos de lechones. Rev Inv Vet Perú: 16: 97-102. doi: 10.15381/rivep.v16i2.1546

20. Li P, Niu Q, Wei Q, Zhang Y, Ma X, Kim S, Lin M, Huang R. 2017. Microbial shifts in the porcine distal gut in response to diets supplemented with Enterococcus faecalis as alternatives to antibiotics. Sci Rep-UK 7: 41395. doi:10.1038/srep41395

21. Mathur S, Singh R. 2005. Antibiotic resistance in food lactic acid bacteria a review. Int J Food Microbiol 105: 281295. doi:10.1016/j.ijfoodmicro.2005.03.008

22. Mejía-Silva W, Rubio-Guillén J, Calatayud-Márquez D, Quintero-Caldera M, Rodríguez-Moreno A. 2007. Evaluación de dos probióticos sobre parámetros productivos en lechones lactantes. Zootecnia Trop 25: 301-306.

23. Ortiz A, Reuto J. 2007. Evaluación de la capacidad probiótica in vitro de una cepa nativa de Saccharomyces cerevisiae. Universitas Scientiarum 13: 138-148.

24. Pantozzi FL, Moredo FA, Vigo GB, Giacoboni GI. 2010. Resistencia a los antimicrobianos en bacterias indicadoras y zoonóticas aisladas de animales domésticos en Argentina. Rev Argent Microbiol 42: 49-52.
25. Piñeros M, Rondón I, Eslava P. 2012. Inmunoestimulantes en teleósteos: probióticos, $\beta$-glucanos y LPS. Orinoquia 16: 46-62.

26. Quemac L. 2014. Evaluación de tres dosis de probiótico (Rhodopseudomonas spp, Lactobacillus spp, Saccharomyces spp) en la alimentación para el engorde de cerdos. Tesis de Ingeniera en Desarrollo Integral Agropecuario. Tulcá, Ecuador: Univ. Politécnica Estatal del Carchi. 62 p.

27. Ross GR, Gusils C, Oliszewski R, Holgado S, González SN. 2010. Effects of probiotic administration in swine. $\mathrm{J}$ Biosci Bioeng 109: 545-549. doi:10.1016/ j.jbiosc.2009.11.007

28. Scott KP. 2002. Cellular and molecular life sciences. The role of conjugative transposons in spreading antibiotic resistance between bacteria that inhabit the gastrointestinal tract. Cell Mol Life Sci 59: 2071-2082.

29. Shu Q, Qu F, Gill H. 2001. Probiotic treatment using Bifidobacterium lactis HN019 reduces weanling diarrhea associated with rotavirus and Escherichia coli infection in a piglet model. J Pediatr Gastroenterol Nutr 33: 171-177.

30. Taras D, Vahjen W, Macha M, Simon O. 2005. Response of performance characteristics and fecal consistency to long-lasting dietary supplementation with the probiotic strain Bacillus cereus var. toyoi to sows and piglets. Arch Anim Nutr 59: 405-417. doi: 10.1080/17450390500353168

31. Taras D, Vahjen W, Simon O. 2007. Probiotics in pigs-modulation of their intestinal distribution and of their impact on health and performance. Livest Sci 108: 229-231. doi: 10.1016/j.livsci.2007.01.075

32. Taras D, Vahjen W, Macha M, Simon O. 2006. Performance, diarrhea incidence, and occurrence of Escherichia coli virulence genes during long-term administration of a probiotic Enterococcus faecium strain to sows and 
piglets. J Anim Sci 84: 608-617. doi: 10.2527/2006.843608x

33. Williams $B A$, Verstegen $M W$, Tamminga S. 2001. Fermentation in the large intestine of single-stomached animals and its relationship to animal health. Nutr Res Rev 14: 207-228. doi: 10.1079/NRR200127
34. Zimmermann JA, Fusari ML, Rossler E, Blajman JE, Romero-Scharpen A, Astesana DM, Olivero CR, et al. 2016. Effects of probiotics in swines growth performance: a meta-analysis of randomised controlled trials. Anim Feed Sci Tech 219: 280-293. doi: 10.1016/ j.anifeedsci.2016.06.021 